\title{
Preconception and the Young Cancer Survivor
}

\author{
Margo C. Grady, M.S., C.G.C.
}

Received: 21 November 2005 / Accepted: 20 April 2006 / Published online: 9 June 2006

(C) Springer Science+Business Media, Inc. 2006

Keywords Preconception $\cdot$ Fertility preservation $\cdot$ Ovarian failure $\cdot$ Pregnancy after cancer

\section{Introduction}

While the diagnosis of cancer is not common in young men and women, cancer survivors younger than 40 are likely to be very concerned about the impact of their cancer on future fertility, their risk of cancer recurrence after treatment and risks to future offspring. It is estimated that 1 in 900 persons between 16 and 44 are cancer survivors [1], and that there are at least 250,000 women age 40 and younger who are breast cancer survivors in the United States. As cancer survival increases, the focus on quality of life issues increases. When questioned, at least $73 \%$ of young breast cancer survivors reported concerns about infertility due to cancer treatment, and $29 \%$ reported that this concern impacted their choice of cancer treatment [2].

\section{Premature ovarian failure}

In women, the options for preserving fertility and risk of ovarian failure depends on the age at the time the cancer is treated, the type and location of cancer, and the specific treatment chosen. Some treatments, such as bone marrow transplantation (BMT) are accompanied by very high rates (>99\%) of ovarian failure [3]. Even for those women who resume normal menstruation after chemotherapy, premature ovarian failure (POF) is not uncommon [4], and the risk of

M. C. Grady, ( $\square)$

Meriter Hospital,

202 S. Park St., Madison, WI 53715

e-mail:mgrady@meriter.com premature ovarian failure is 8 -fold higher in cancer survivors [5]. The risk of POF increases with the woman's age, with less than $20 \%$ of women under 30 years experiencing POF, whereas most women older than 40 will become menopausal after chemotherapy [6].

POF and menopause are dependent on the type of therapy used. Rates of ovarian failure range from $15 \%$ for acute myelocytic leukemia (AML) to $50 \%$ for breast cancer, with the overall rate of ovarian failure of $34 \%$ [4]. The chemotherapy agents with the highest risk for ovarian failure were the alkylating agents. The higher the dose of these drugs, the higher the likelihood of ovarian failure.

Ovarian tissue damage is well documented with the use of radiation therapy. Radiotherapy is used for treatment of several cancer types affecting pre-menopausal women. The degree of damage to ovarian tissue is related to a woman's age, the total dose of radiation used, and the number of exposures. In one study, when the same total dose of radiation was delivered, exposure to multiple fractionated doses versus a single dose resulted in less damage to the ovaries [4]. When used in conjunction with chemotherapy, the risk of POF increases. Byrne et al. assessed the risk of premature ovarian failure in childhood cancer survivors and found the relative risk of early menopause was 9.2 for those treated with chemotherapy alone, and 27 for those treated with radiation plus chemotherapy [5].

\section{Fertility preservation techniques}

Although an increasing number of techniques have become available for the preservation of fertility, many are experimental and have not been tested in randomized controlled trials. Table 1 reviews the types of options available and their benefits and limitations. 
Table 1 Fertility preservation options for women with malignancies

\begin{tabular}{lll}
\hline Method & Benefits & Limitations \\
\hline In vitro Fertilization (IVF) & Most successful, widespread & Delay of therapy, use of fertility medications \\
Frozen oocytes & No partner is needed & Difficult to preserve \\
Ovarian tissue preservation & Less tissue damage from freezing, can be used & Experimental \\
& in pre-menarchal and young women & Limited to women having radiation therapy \\
Transposition of ovarian tissue & Less radiation exposure & Adequate prospective studies needed \\
Pharmacological protection & Limit damage to ovarian tissue & \\
\hline
\end{tabular}

\section{In vitro fertilization (IVF)}

The best-studied and most successful option for preserving fertility in female cancer patients is ovarian stimulation followed by IVF and embryo cryopreservation. Ovarian stimulation needs to occur prior to cancer therapy because treatment can adversely affect ovarian reserve and response. The pregnancy rate with frozen embryos is lower compared to fresh IVF embryo transfer. A meta-analysis by the American Society for Reproductive Medicine reviewed over 88000 cycles of assisted reproductive technology (ART) treatment. When fresh oocytes were used, a delivery rate of $41.8 \%$ was found in more than 6500 cycles analyzed, compared to a delivery rate of $18.6 \%$ for frozen embryos [7].

One concern about ovarian stimulation with IVF is that it requires a delay of therapy, which could impact growth rate of tumors and survival rates. In addition, concerns have been voiced that the medications used to stimulate ovarian production could impact on estrogen-receptor positive tumors such as breast carcinoma. Anti-estrogens, such as tamoxifen, or aromatase inhibitors, (e.g. letrozole), have both been used in ovarian stimulation in breast cancer patients to maintain low estradiol levels during IVF cycles.

\section{Frozen oocytes}

Frozen oocytes are more difficult to preserve, resulting in a lower survival, fertilization and pregnancy rates. Damage is in part due to ice formation during the freezing process. Unfertilized frozen oocytes have a low success rate for live birth (2\% per oocyte) [8], although the rates are now increasing. Rapid freezing of eggs by vitrification limits damage, which improves oocyte survival. Using intracytoplasmic sperm injection (ICSI) increases the fertilization rate even further.

\section{Ovarian tissue cryopreservation}

For pre-menarchal girls, young women with POF, women that cannot delay therapy or those in whom high doses of fertility medications are contraindicated, ovarian tissue cryop- reservation is offered in some centers. Unlike frozen oocytes, primordial follicles in ovarian tissue are smaller and have less follicular fluid, and therefore may be at less risk from damage from ice crystals that form during freezing. It is hoped that transplanting thawed ovarian tissue will result in the recovery of fertility. Transplantation should be used with caution in women with ovarian or metastatic cancer, to prevent transferring malignant cells back after treatment. This treatment is still considered experimental. Although success in animals has been demonstrated, transplantation of cryopreserved ovarian tissue has resulted in only two livebirths $[9,10]$.

\section{Ovarian transposition}

For women having pelvic radiation, transposition of ovarian tissue outside of the field of radiation successfully preserves ovarian function. The radiation dose to the transposed ovary is approximately $5-10 \%$ the level of the non-transposed ovary [11].

\section{Pharmacological protection}

To limit or prevent chemotherapy-induced POF, some centers use gonadatropin releasing hormone $(\mathrm{GnRH})$ agonists to reduce gonadatropins. This results in mimicking a prepubertal state, because the ovary appears to be less sensitive to gonadotoxic drugs prior to menarche. Because dividing cells are more susceptible to damage from cytotoxic agents, ovarian follicular cells are vulnerable. The mechanism of protection by GnRH agonists is unknown. One prospective clinical case series found that 70 of 75 women (93\%) co-treated with GnRH agonists and chemotherapy resumed cyclic ovarian function verses 38 of 82 women (46\%) treated with chemotherapy alone [12]. Adequately controlled prospective studies still are required, although data from animal studies are promising. Anti-apoptotic agents, such as sphingosine-1-phosphate (SIP), have prevented radiationinduced oocyte loss in mice [13]. However, since apoptosis is needed for the treatment of cancer, this method faces some obstacles for implementation. 


\section{Male fertility}

In men, sperm banking is routine and should be offered to all men prior to undergoing cancer treatment. When combined with intracytoplamic sperm injection (ICSI), sperm banking should preserve fertility for almost all males. There is no increased risk for birth defects when sperm is banked prior to therapy $[14,15]$. Even in azoospermic males, testicular sperm extraction (TESE) with ICSI has resulted in successful pregnancies. By 2005, 11 of 29 men with azoospermia that had used TESE with ICSI had achieved a pregnancy which resulted in a livebirth [16]. For pre-pubertal males, sperm banking is not available because there is no spermatogenesis. The possibility of cryobanking of testicular tissue is being explored [16, 17]. An increase in sperm aneuploidy is seen up to 18 months post-chemotherapy, so it has been suggested that a delay of conception for 12-18 months after therapy is completed is reasonable [18].

\section{Pregnancy after cancer}

Once a woman has recovered from cancer treatment, no clear guidelines exist about the length of time to wait before attempting to become pregnant.

\section{Effect of cancer treatment on pregnancy}

Once a cancer survivor achieves pregnancy, her health and the fetal health may be adversely affected by her prior treatment for cancer. Clinically significant heart disease occurs in $0.5-1 \%$ of women treated with anthracycline-based chemotherapy drugs, and may not develop until months or years after therapy is completed [19]. Also, women who have radiation to the left side of the abdomen or chest are at risk for heart damage, which could worsen during pregnancy or labor. Women with radiation to the abdomen or pelvic area or total body radiation are at increased risk for miscarriage, preterm birth and delivery of low birthweight infants [20]. A report from the Childhood Cancer Survivor Study (CCSS) did not show any increase in adverse pregnancy outcomes in women treated with most chemotherapy agents. However, an increased risk of stillbirth in women treated with abdominal radiation for Wilm's tumor has been noted [21].

Women who have completed treatment for breast cancer are often placed on selective estrogen receptor modulators (SERMs) such as tamoxifen to reduce the incidence of a second cancer. However, tamoxifen use during pregnancy is to be avoided because it is potentially teratogenic.

\section{Effect of pregnancy on cancer}

Some women are concerned that the hormonal changes from pregnancy may increase the risk of cancer recurrence, but pregnancy after breast cancer management does not increase the risk of cancer recurrence or cancer-related death $[22$, 23]. However, women who are carriers of $B R C A I$ or $B R C A 2$ germline mutations and who have had a full-term pregnancy are at significantly higher risk of developing breast cancer by age 40 than mutation carriers who do not have children [24]. The risk for breast cancer is increased by $70 \%$ in the first 2 years after pregnancy for $B R C A 2$ carriers, but not $B R C A 1$ carriers [25].

\section{Risk to offspring}

Since cancer treatment by radiation and many chemotherapy agents are known to cause DNA mutations, cancer survivors are often concerned that the treatment may increase the risk for birth defects, genetic conditions or cancer in their offspring. Several studies have found no increased risk of congenital abnormalities in the offspring of cancer survivors. Epidemiologic data from Japanese survivors of the atomic bomb and their children did not show any evidence for new mutations. The CCSS also did not find any link between radiation therapy or chemotherapy for childhood cancer and genetic diseases or birth defects [26, 27].

\section{Risk of cancer in offspring}

No increased risk for childhood cancer was identified in the offspring of childhood cancer survivors, but additional follow-up is needed [26]. In rare instances, the childhood cancer may be hereditary, with a risk of passing on the mutated gene to the offspring. Some cancers, such as breast, colon or ovarian cancer, are more likely to be hereditary when the cancer occurs at an earlier age. For women with breast cancer diagnosed prior to age 40 and no other family history of early breast cancer, the risk of a mutation in one of the hereditary breast cancer genes, $B R C A I$ or $B R C A 2$ is estimated to be at least $13 \%$ [28].

\section{Summary}

All women should be counseled about the impact of cancer treatment on fertility prior to undergoing chemotherapy, ovarian surgery or BMT, and offered fertility preservation options, of which ovarian stimulation with IVF is the most successful. Once women achieve pregnancy, the risk for birth defects or genetic conditions is not increased because of 
either radiation or chemotherapy. Because treatment options may increase the risk for adverse pregnancy outcome, review of therapy records is essential for determining potential risk to the pregnancy. For most young cancer survivors, fertility can be preserved and a successful pregnancy achieved, but discussing these issues prior to treatment is essential.

Acknowledgement I would like to thank Dr. Herschel W. Lawson for his critical commentary.

\section{References}

1. Toren A, Rechavi G, Ramot B. Pediatric cancer: environmental and genetic aspects. Pediatric Hematol Oncol 1996;13:319-31.

2. Partridge AH, Gelber S, Peppercorn J, et al. Web Based Survey of fertility issues in young women with breast cancer. J Clin Oncol 2004;22:4174-83.

3. Mertens AC, Ramsay NK, Kouris S, Neglia JP. Patterns of gonadal dysfunction following bone marrow transplantation. Bone Marrow Transplant 1998;22:345-50.

4. Meirow D, Nugent D. The effects of radiotherapy and chemotherapy on female reproduction. Hum Reprod Update 2001;7:535-43.

5. Byrne J, Fears TR, Gail MH, et al. Early menopause in longterm survivors of cancer during adolescence. Am J Obstet Gynecol 1992;166:788-93.

6. Partridge AH, Burstein HJ, Winer EP. Side effects of chemotherapy and combined chemohormonal therapy in women with early-stage breast cancer. J Natl Cancer Inst Monogr 2001;30:135-142.

7. American Society for Reproductive Medicine. Society for Assisted Reproductive Technology Registry. Assisted reproductive technology in the United States: 1999 results generated from the American Society for Reproductive Medicine/Society for Assisted Reproductive Technology Registry. Fertil Steril 2002;78:918-31.

8. Gosden RG. Prospects for oocyte banking and in vitro maturation. J Natl Cancer Inst Monogr 2005;34:60-3.

9. Donnez J, Dolmans MM, Demylle D, et al. Livebirth after orthotopic transplantation of cryopreserved ovarian tissue. Lancet 2004;364:1405-10.

10. Meirow D, Levron J, Eldar-Geva T, et al. Pregnancy after transplantation of cryopreserved ovarian tissue in a patient with ovarian failure after chemotherapy. N Engl J Med 2005;353:318-21.

11. Howell SJ, Shalet SM. Fertility preservation and management of gonadal failure associated with lymphoma therapy. Curr Oncol Rep 2002;4:443-52.

12. Blumenfeld Z, Eckman A. Preservation of fertility and ovarian function and minimization of chemotherapy-induced gonadotoxicity in young women by GnRH-a. J Natl Cancer Inst Monogr 2005;34:40-3.
13. Morita Y, Perez GI, Paris F, et al. Oocyte apoptosis is suppressed by disruption of the acid sphingomyelinase gene or by sphingosine1-phosphate therapy. Nat Med 2000;6:1109-14.

14. Agarwal A, Ranganathan P, Kattal N, Pasqualotto F, Hallak J, Khayal S, Mascha E. Fertility after cancer: a prospective review of assisted reproductive outcome with banked semen specimens. Fertil Steril 2004;81:342-8.

15. Green DM, Whitton JA, Stovall M, et al. Pregnancy outcome of partners of male survivors of childhood cancer: a report from the Childhood Cancer Survivor Study. J Clin Oncol 2003;21:71621.

16. Shin D, Lo KC, Lipshultz. Treatment options for the infertile male with cancer. J Natl Cancer Inst Monogr 2005;34:48-50.

17. Tournaye H, Goossens E, Verheyen G, Frederickx V, De Block G, Devroey P, Van Steirteghem A. Preserving the reproductive potential of men and boys with cancer: current concepts and future prospects. Hum Reprod Update 2004;10:525-32.

18. De Mas P, Daudin M, Vincent MC, Bourrouillou G, Calvas P, Mieusset R, Bujan L. Increased aneuploidy in spermatozoa from testicular tumour patients after chemotherapy with cisplatin, etoposide and bleomycin. Hum Reprod 2001;16:1204-8.

19. Shapiro CL, Recht A. Drug therapy: side effects of adjuvant treatment of breast cancer. N Engl J Med 2001;344: 1997-2008.

20. Critchley HOD, Wallace WHB. Impact of cancer treatment on uterine function. J Natl Cancer Inst Monogr 2005;34: 64-8.

21. Green DM, Whitton JA, Stovall M, et al. Pregnancy outcome of female survivors of childhood cancer: a report from the Childhood Cancer Survivor Study. Am J Obstet Gynecol 2002;187:107080 .

22. Velentgas P, Daling JR, Malone KE, Weiss NS, Williams MA, Self SG, Mueller BA. Pregnancy after breast carcinoma: outcomes and influence on mortality. Cancer 1999;85:2424-32.

23. Kroman N, Jensen M-B, Melbye M, Wohlfahrt J, Mouridsen HT. Should women be advised against pregnancy after breast-cancer treatment? Lancet 1997;350:319-22.

24. Jernstrom H, Lerman C, Ghadirian P, et al. Pregnancy and risk of early breast cancer in carriers of BRCA1 and BRCA2. Lancet 1999;354:1846-50.

25. Cullinane CA, Lubinski J, Neuhausen SL, et al. Effect of pregnancy as a risk factor for breast cancer in BRCA1/BRCA2 mutation carriers. Int J Cancer 2005; 117:988-91.

26. Boice JD, Tawn EJ, Winther JF, et al. Genetic Effects of radiotherapy for childhood cancer. Health Phys 2003;85:65-80.

27. Green DM, Fiorello A, Zevon MA, Hall B, Seigelstein N. Birth defects and childhood cancer in offspring of survivors of childhood cancer. Arch Pediatr Adolesc Med 1997;151:379-83.

28. Frank TS, Deffenbaugh AM, Reid JE, et al. Clinical characteristics of individuals with germline mutations in BRCA1 and BRCA2: analysis of 10,000 individuals. J Clin Oncol 2002;20:148090. 